\title{
Ranking Participants in Tournaments by means of Rating Functions
}

\author{
Miguel Brozos-Vázquez \\ Dept. of Geometry and Topology \\ Universidade de Santiago de Compostela
}

\section{José Carlos Díaz-Ramos}

Dept. of Mathematics

University College Cork

\section{Marco Antonio Campo-Cabana}

Dept. of Applied Mathematics

Universidade de Santiago de Compostela

\section{Julio González-Díaz*}

Kellogg School of Management

Northwestern University

\begin{abstract}
In this paper we bring a novel approach to the theory of tournament rankings. We combine two different theories that are widely used to establish rankings of populations after a given tournament. First, we use the statistical approach of paired comparison analysis to define the performance of a player in a natural way. Then, we determine a ranking (and rating) of the players in the given tournament. Finally, we show, among other properties, that the new ranking method is the unique one satisfying a natural consistency requirement.
\end{abstract}

\section{Introduction}

When there is a competition among the members of a population, the fundamental problem is to rank these members according to their strength 1 In certain cases this confrontation takes the form of a tournament in which contestants play against themselves obtaining a certain score in each match. The aim is to determine a final ranking after all the matches have been played. Because of the wide range of applications of ranking theory, the latter problem has already been widely studied in management science, economic theory and statistics. The books by Moon (1968) and Laslier (1997) and the paper Iqbal et al. (1986) discuss several applications of the theory of tournament rankings.

Formally, a ranking of a population $N$ is a complete, reflexive and transitive relation on $N$. To fix notation, we use the word rating when we have a cardinal ranking, that is, not only do we have an ordering of the contestants, but also a measure of the intensities of the differences among them.

In this paper, we define a measure of the strength exhibited by the players of a given tournament that we call performance. More specifically, we assume that there is a distribution function that governs the random process associated with the competitive environment in which the given tournament takes place. Within this setting, the performance of a player

\footnotetext{
* Corresponding author: Kellogg School of Management (CMS-EMS), Northwestern University. 5100 Leverone Hall, 2001 Sheridan Road. Evanston, IL 60208-2014. Phone number: +1 847-467-1745. Fax: +1 847-491-2530. E-mail address: julio@northwestern.edu

${ }^{1}$ We usually refer to the members of our population as contestants or players, but they may also be other objects such as scientific journals, political options or products to be tested.
} 
is naturally defined as a function of his results and the strength of his opponents. Then, we define a new ranking method, the recursive performance, and show that it is the unique one that is consistent with the notion of performance.

The most natural attempt to associate a ranking to a given tournament is to use the scores ranking. That is, rank the players according to their total scores. Rubinstein (1980) provides an axiomatic characterization of the scores ranking. Nonetheless, it is often the case that several players have the same score and, hence, in most scenarios this ranking method does not provide an ordering of the players. On the other hand, most ranking methods determine the ranking of a player according to the results obtained in the tournament and the strength of the opponents the player has played against, being this last feature missing when using the scores ranking 2 One widely used ranking method that takes the previous considerations into account is the minimum violations ranking, where one violation consists of two players whose relative ranking differs from the one induced by their result against each other. This ranking method is discussed, for instance, in Goddard (1983) and Iqbal et al. (1986). However, Stob (1985) is quite critical with it and sticks up for the statistical approach of paired comparison analysis initiated in Zermelo (1929) for chess tournaments and rediscovered by Bradley and Terry (1952).

In Bradley and Terry (1952), each player $i$ is assumed to have a strength parameter $r_{i}$. It is also assumed that there exists a distribution $F$ such that $F\left(r_{i}, r_{j}\right)$ is the probability that $i$ beats $j$. The objective is to fix a distribution $F$ that properly fits the available data and then, using statistical tools, calculate the most likely values of the strength parameters $r_{i}$. Once these values are calculated, they may be used to define a rating for each player. As compared with the minimum violations ranking, Stob (1985) emphasizes that this approach endogenizes the relevance that must be given to the different victories (losses) of the players, a feature that we also consider very desirable. Two classic references within this framework are Kendall and Smith (1940) and David (1988).

Finally, a third approach comes from economic theory, where it is often the case that an axiomatic approach is taken to determine a ranking of the population $N$. First, it is assumed that there is a matrix containing the relevant information about the paired results of the different players; this matrix is usually referred to as the tournament matrix. Then, a ranking method is defined as a function that ascribes a ranking to each tournament matrix. Next, the properties of the different methods are studied. Finally, a ranking method, whose properties are suitable for a given competitive environment, is chosen. To deepen into the economic literature on rankings refer to Rubinstein (1980), Liebowitz and Palmer (1984), Amir (2002), Palacios-Huerta and Volij (2004), and Slutzki and Volij (2005).

In this paper we consider a competitive environment with an associated function $F$ that describes the underlying random process. This function is determined from the data of the historical confrontations of the players in a population. Then, we assume that we have one more tournament and we want to rank the players of that tournament according to their results. Next, we bring together two widely used ideas. On the one hand, our ranking method, the recursive performance, is defined using a recursive formula that resembles the Liebowitz-Palmer method (Liebowitz and Palmer, 1984) and other similar methods (Palacios-Huerta and Volij, 2004) studied in economic theory. On the other hand, our recursive formula uses the rating function $F$, so basic in the statistical approach to paired comparison analysis. Moreover, we show one property of our ranking method that is crucial for its applicability. Namely, our ranking method is robust with respect to the estimation of the function $F$, that is, small changes in the function $F$ lead to small changes

\footnotetext{
${ }^{2}$ Indeed, this is the reason why the scores ranking is mainly used in round-robin tournaments, where each player faces the same opponents.
} 
in the proposed rating. Finally, concerning the computation of our ranking method, we show that it reduces to solving a linear system.

Within the literature in which this paper is enclosed, there is a family of tournaments that has received special attention: the round-robin tournaments. In these tournaments each player plays exactly once against any other player. The minimum violations ranking is essentially thought for this class of tournaments. Stob (1985) showed that, under quite general assumptions, the ranking methods defined using the approach of Zermelo (1929) lead to the same ordering as the scores ranking. We briefly discuss the behavior of our approach in round-robin tournaments at the end of Section 4.

As an immediate application, the results obtained in this paper can be applied to define new tie-breaking rules for disciplines such as chess and Othello 3 In our opinion, these new tie-breaking rules improve the existent ones (see Sections 4 and 5 for details).

We briefly describe the contents of this paper. In Section 2 we present the concept of tournament and comment on the assumptions used throughout this paper. In Section 3 we formally define and discuss the notion of performance. Section 4 is the core of our study; we introduce the so-called recursive performance and give examples of tournaments in which it might be immediately applied. In Section 5 we discuss two properties of the recursive performance ranking method: robustness and consistency. Finally, in Section 6 we prove the results presented in Sections 4 and 5 .

\section{Basic Definitions: Tournaments}

We have a competitive environment in which confrontations between the different players of a population take place along time. For such an environment, there is a rating function $F$ that accurately describes the probabilities associated with the different results of each match between any two given players. This rating function is such that, given two players $i$ and $j$ with strength parameters $r_{i}$ and $r_{j}$, the probability that $i$ beats $j$ is $F\left(r_{i}, r_{j}\right)$. Thus, $F\left(r_{i}, r_{j}\right)=1-F\left(r_{j}, r_{i}\right)$, We refer to the strength parameters $r_{i}$ as ratings.

We work within the linear paired comparison model (David, 1988). More specifically, we assume that there is a strictly increasing continuous distribution function $F_{l}: \mathbb{R} \rightarrow(0,1)$ such that $F\left(r_{1}, r_{2}\right)=F_{l}\left(r_{1}-r_{2}\right)$, that is, the result of a game between any two players depends only on their rating difference. The probability that $i$ beats $j$ goes to 1 as $r_{i}-r_{j}$ grows and the probability that $i$ beats $j$ is positive regardless of the rating difference. Moreover, since $F\left(r_{i}, r_{j}\right)=1-F\left(r_{j}, r_{i}\right), F_{l}$ is symmetrically distributed about zero. Also, note that the function $F_{l}^{-1}$ is well defined.

The result of a confrontation between two players $i$ and $j$ may be not only a win or a loss but any pair $\left(a_{i}, a_{j}\right)$ with $a_{i}, a_{j} \geq 0, a_{i}+a_{j}=1$. Within this scenario we might interpret $F\left(r_{i}, r_{j}\right)$ as the expected score of player $i$ when facing player $j$.

We denote by $\mathcal{M}_{k \times l}$ the vector space of real $k \times l$ matrices. A tournament is a pair $(N, A)$, where $N=\{1, \ldots, n\}$ is the set of players and $A \in \mathcal{M}_{n \times n}$ is the tournament matrix. The matrix $A$ is non-negative and its main diagonal consists of zeros. The entry $A_{i j}$ contains the score achieved by player $i$ in his confrontations against player $j$. Note that we do not restrict the non-zero entries in our matrix $A$ to be natural numbers as in Slutzki and Volii (2005). To each tournament $(N, A)$ we can naturally associate a (symmetric) matches matrix $M(A):=A+A^{t}$, i.e., $M(A)_{i j}$ is the number of matches between $i$ and $j$. For

\footnotetext{
${ }^{3}$ Remarkably, ties are always present in tournaments in which pairings are drawn following the Swiss pairing system, which is, along with the round-robin system, the most widely used in these disciplines.

${ }^{4}$ For instance, chess and Othello use rating systems based on functions that have already been widely tested.
} 
notational simplicity, when no confusion arises we denote $M(A)$ by $M$. Since the $n$ players participate in the tournament, each row of $M$ must have a nonzero entry. For each player $i$, let $m_{i}:=\sum_{j=1}^{n} M_{i j}$ denote the total number of matches played by $i$. Let $r \in \mathbb{R}^{n}$ be a vector of exogenously given ratings of the players in the tournament $(N, A)$. We refer to $r$ as the vector of initial ratings.

Given a tournament $(N, A)$ and a vector of ratings $r$, we define the total strength of the tournament by $\sigma^{r}:=\sum_{i=1}^{n} m_{i} r_{i}$, that is, the contribution of each player to the total strength of the tournament is weighted by the number of matches he has played. Similarly, we define the average strength of the tournament by $\bar{\sigma}^{r}:=\sigma^{r} / \sum_{i=1}^{n} m_{i}$. Note that, if all the players have played the same number of games, the average strength of the tournament is just the average of the vector of initial ratings.

Summarizing, the primitives of our model are a tournament $(N, A)$, a rating function $F$, and a vector of initial ratings $r$. In this paper we present a ranking and rating method for the tournament in question. Our ranking method reallocates among the players the total strength of the tournament, $\sigma^{r}$, with two important features: first, the proposed ranking does not depend on $r$ and, second, the difference between the proposed ratings for any two players is also independent of $r$. That is, our method is endogenous to $(N, A)$ and the vector $r$ is used just for the sake of exposition.

Since the ranking method we define in this paper is anonymous, the labels chosen for indexing the players are irrelevant. Based on this fact, two tournaments that are equal up to labeling are said equivalent. We make this definition precise. Denote by $L_{\alpha \beta} \in \mathcal{M}_{n \times n}$ the transposition matrix that swaps the $\alpha$ th and $\beta$ th entries of a vector. A transposition matrix satisfies $L_{\alpha \beta}^{-1}=L_{\alpha \beta}$ and, given $B \in \mathcal{M}_{n \times n}$, the product $L_{\alpha \beta} B$ is the same matrix $B$ but with rows $\alpha$ and $\beta$ interchanged. Similarly, $B L_{\alpha \beta}$ interchanges columns $\alpha$ and $\beta$ of $B$. The group $\Pi_{n}$ generated by the composition of transposition matrices $L_{\alpha \beta}$ is isomorphic to the group of permutations of $n$ elements. Given two populations $N$ and $N^{\prime}$ with $n$ players, we say that two tournaments $(N, A)$ and $\left(N^{\prime}, A^{\prime}\right)$ are equivalent if there exists $L \in \Pi_{n}$ such that $A=L A^{\prime} L^{t}$. Note that the latter also implies that $M=L M^{\prime} L^{t}$. Since for each $L \in \Pi_{n}$ we have $L^{-1}=L^{t}$, two equivalent tournaments have similar tournament matrices and similar matches matrices.

A matrix $B \in \mathcal{M}_{n \times n}$ is block diagonal, respectively block anti-diagonal, if

$$
B=\left(\begin{array}{l|l}
* & 0 \\
\hline 0 & *
\end{array}\right), \text { respectively } B=\left(\begin{array}{l|l}
0 & * \\
\hline * & 0
\end{array}\right) .
$$

Note that, given a tournament $(N, A), A$ is block diagonal (anti-diagonal) if and only if $M$ is block diagonal (anti-diagonal). We assume that our tournaments satisfy the following two natural assumptions:

A1. The tournament $(N, A)$ is not equivalent to a tournament $\left(N^{\prime}, A^{\prime}\right)$ such that $A^{\prime}$ is block diagonal.

If the tournament matrix $A^{\prime}$ is block diagonal, the tournament has an internal division: there are two disjoint subsets of players such that none of the players of one set has played against anyone of the other set. This is a standard assumption in the ranking's literature since each block may be considered as the matrix of an independent tournament.

A2. The tournament $(N, A)$ is not equivalent to a tournament $\left(N^{\prime}, A^{\prime}\right)$ such that $A^{\prime}$ is block anti-diagonal.

If $A^{\prime}$ is block anti-diagonal, the tournament may be considered as a team-tournament. There are two disjoint subsets (teams) such that the players of each team have played only 
against the players of the other, but not among themselves. Although similar to A1, this property is more subtle and has different implications. In this case, in order to calculate the strength of the players of one team, we need the strength of the players of the other team that can only be calculated using the strength of the players in the first team. This cyclic feature of team-tournaments is the reason why, if $\mathrm{A} 2$ is not met, the iterative method we describe in Section 4 does not necessarily converge.

\section{The Notion of Performance}

Let $(N, A)$ be a tournament. The vector of average scores, $s$, is defined by $s_{i}:=\sum_{j} A_{i j} / m_{i}$. Hereafter, we assume that $s \in(0,1)^{n}$. We define $D^{M}:=\operatorname{diag}\left(m_{1}, \ldots, m_{n}\right) \in \mathcal{M}_{n \times n}$ and $\bar{M}:=\left(D^{M}\right)^{-1} M \in \mathcal{M}_{n \times n}$, that is, $\bar{M}_{i j}=M_{i j} / m_{i}$ is the number of confrontations between $i$ and $j$ divided by the total number of matches played by $i$.

Let $r \in \mathbb{R}^{n}$ be the vector of initial ratings and let $F$ be the distribution function of the linear paired comparison model. The vector of $r$-performances, $p^{r} \in \mathbb{R}^{n}$, is defined as

$$
p^{r}:=\bar{M} r+c, \quad \text { where } c_{i}:=F_{l}^{-1}\left(s_{i}\right) .
$$

Note that $(\bar{M} r)_{i}$ coincides with the average rating of the opponents of player $i$. Hence, the $r$-performance of player $i$ is the unique rating $p_{i}^{r}$ such that $F\left(p_{i}^{r},(\bar{M} r)_{i}\right)=s_{i}$. Then, we might say that $s_{i}$ is $i$ 's expected score against a player of rating $(\bar{M} r)_{i}$ if and only if $i$ has a rating $p_{i}^{r}$. This justifies the name performance.

The vector $p^{r}$ depends on the tournament and the rating function associated with the competitive environment which the tournament belongs to. Nevertheless, it also depends on the vector of initial ratings, which is exogenous to the tournament. On the other hand, the $r$-performance of player $i$ takes into account the strengths of his opponents; however we consider that also the opponents of the opponents of $i$ should be used to calculate the strengths of the opponents of $i$, and the $r$-performances do not do so. We devote Section 4 to address these two problems.

Example 1. The World Chess Federation (FIDE) has an official rating of players called Elo. Elo's formula considers the distribution $F_{l}$ given by $F_{l}(\lambda)=1 /\left(1+10^{-\frac{\lambda}{400}}\right)$. Hence, the $r$-performance of player $i$ in a tournament is defined as $p_{i}^{r}=(\bar{M} r)_{i}-400 \log _{10}\left(1 / s_{i}-1\right)$, that is, the average of the Elos of his opponents plus a correcting factor depending on the percentage of points achieved by the player. Remarkably, this is one of the tie-breaking rules recommended by the FIDE for chess tournaments.

The assumption $s \in(0,1)^{n}$ is needed in order to define the vector of performances correctly, but this situation holds in most tournaments. Indeed, the $r$-performance ranking method is already used as a tie breaking rule for chess tournaments, as we have just seen. The idea of this method is to use the strengths of the opponents of the players to define the rankings. Note that the vector $r$ is the "historical" strength of the players whereas the vector $c$ is, essentially, the score of each player in the tournament. Hence, $p^{r}$ measures the results of a player in relation to the strength of his opponents.

\section{Recursive Performance}

In the definition of $r$-performance, the vector $c$ depends crucially on the rating function $F$ and, although our notation does not make this dependence explicit, the rating function keeps being an essential element of this paper. 
The ranking associated with the vector of $r$-performances is not a bad ranking for the tournament, but it heavily depends on the initial ratings $r_{1}, \ldots, r_{n}$. The latter measure the historical strength of the players, which might be different from the strength exhibited by the players in the tournament. Moreover, in the paired comparison literature, these ratings are often calculated using the method of maximum likelihood, and thus, they are subject to statistical errors.

The objective of this section and the next one is to formally introduce a new ranking method, the recursive performance, and discuss some of its properties. Refer to Section 6 for the technical results and their corresponding proofs.

As we have already discussed in Section 3 , even though $\left(p^{r}\right)_{i}$ is a better indicator of the strength of $i$ in the given tournament than his initial rating, using the vector $p^{r}$ as a ranking method has relevant shortcomings. Nonetheless, it is natural to calculate a new performance by replacing the initial ratings with the vector of performances. This would have two main effects. Namely, it would shade the dependence on $r$ and, given a player $i$, the new rating would take into account, not only the opponents of $i$, but also their results (i.e., using the opponents of the opponents of $i$ as well). This suggests the iterative method $p^{(0)}:=p^{r}(=\bar{M} r+c), p^{(l+1)}:=p^{p^{(l)}}\left(=\bar{M} p^{(l)}+c\right)$. Unfortunately, this method does not necessarily converge. On the other hand, the total strength of the tournament, $\sigma^{r}$, is not preserved by the $r$-performance, that is, $\sum_{i} m_{i} r_{i} \neq \sum_{i} m_{i} p_{i}^{r}$ in general. The inflation or deflation factor turns out to be $\sum_{i} m_{i} c_{i}$. By distributing this factor among the players, we define a new iterative method that does preserve the total strength of the tournament.

Let $e \in \mathbb{R}^{n}$ be the vector $e=(1, \ldots, 1)$. Consider the following rescaling of $c$,

$$
\hat{c}:=c-\left(\frac{\sum_{i} m_{i} c_{i}}{\sum_{i} m_{i}}\right) e
$$

which we discuss below. We define the iterated performance as the iterative method

$$
\begin{aligned}
\hat{p}^{(0)} & :=\bar{M} r+\hat{c} \\
\hat{p}^{(l)} & :=\bar{M} \hat{p}^{(l-1)}+\hat{c}, \quad l \in \mathbb{N} .
\end{aligned}
$$

At each step $l$, this iterative method gives the same rating as the previous one up to a constant proportional to $e$ and, hence, the two proposed rankings are always the same. This is proved in the following lemma.

Lemma 1. For each $l \in \mathbb{N}, p^{(l)}-\hat{p}^{(l)}=(l+1)\left(\frac{\sum_{i} m_{i} c_{i}}{\sum_{i} m_{i}}\right) e$.

Proof. Since by definition of $\bar{M}, \bar{M} e=e$, the result follows by an induction argument.

In Section 6 we prove that, under our assumptions, the iterated performance converges. More specifically, we have the following result.

Theorem 2. Let $(N, A)$ be a tournament satisfying assumptions A1 and A2 and such that $\bar{A}_{i j} \in(0,1)$ whenever $M_{i j} \neq 0$. Let $r \in \mathbb{R}^{n}$ be a vector of initial ratings and $F_{l}$ the distribution of the linear paired comparison model. Then the iterated performance converges.

Motivated by Theorem 2, we define the main concept of this paper, the recursive performance, as the limit of the iterated performance: $\hat{p}:=\lim _{l \rightarrow \infty} \hat{p}^{(l)}$. Taking limits in the equality $\hat{p}^{(l)}=\bar{M} \hat{p}^{(l-1)}+\hat{c}$, we get that $\hat{p}$ is a solution of the linear system

$$
(I-\bar{M}) x=\hat{c}
$$


where $I \in \mathcal{M}_{n \times n}$ is the identity matrix. If A1 holds, by Theorem [5 (iii) below the matrix $I-\bar{M}$ has rank $n-1$. Then, since $\bar{M} e=e$, the whole set of solutions of (1D) is given by $\hat{p}+\operatorname{span}\{e\}$. The different solutions of (1) arise from different initial vectors of ratings $r$. It is important to note that all the solutions propose the same ranking.

Following the previous discussion, even if A2 does not hold, we can unambiguously associate a ranking to each linear system (1) as far as A1 is met.

Example 2. An ideal chess-like tournament is a tournament in which all the players play the same number of rounds, say $k$ 每 Thus, for an ideal chess-like tournament $(N, A)$ we have $D^{M}=k I$ and, hence, $\bar{M}=M / k$. By Corollary 7 below, $\sum_{i} m_{i} \hat{p}_{i}^{(l)}=\sum_{i} m_{i} r_{i}=\sigma^{r}$ for all $l \in \mathbb{N}$. Since $m_{i}=k$ for all $i \in N$, in an ideal chess-like tournament we have $\sum_{i} \hat{p}_{i}^{(l)}=\sum_{i} r_{i}$. This shows that, using $\hat{c}$ instead of $c$, we adjust the vectors after each iteration to ensure that the sum of the ratings after each step remains constant. The average strength of an ideal tournament, $\bar{\sigma}^{r}$, coincides with the average of the components of $r$. In each iteration the method proposes a way to divide the total strength of the tournament, $\sigma^{r}$, among the players. That is, by working with $\hat{c}$ instead of $c$, we avoid inflation or deflation in the vectors of iterated performances. Since the recursive performance is the limit of such vectors, it also provides a way of dividing the total strength of the tournament among the players. In a general tournament, the same property holds, but in this case the average strength of the tournament is calculated as a weighted average (by the $m_{i}$ 's) of the components of $r$.

Example 3. A round-robin tournament $(N, A)$ is a tournament in which all the players have played exactly once against each other. That is, $M_{i j}=1$ if $i \neq j$ and $M_{i i}=06$ Within this family of tournaments, the ranking proposed by the recursive performance has an appealing feature: it coincides with the ranking proposed by the vector of average scores (the standard scores ranking method). To see this, let $x$ be a solution of the system (1). Then, the claim follows from the equality $\hat{c}_{i}-\hat{c}_{j}=x_{i}-\sum_{k \neq i} \frac{x_{k}}{(n-1)}-x_{j}+\sum_{k \neq j} \frac{x_{k}}{(n-1)}=\frac{n}{n-1}\left(x_{i}-x_{j}\right)$. This is not surprising since the ranking proposed by the recursive performance uses both the scores of the players and the scores of the opponents, but all the players have the same opponents.

\section{Main Properties: Robustness and Consistency}

Since all the solutions of (1) propose the same ranking, the recursive performance ranking method does not depend on the vector of initial ratings. Moreover, the recursive performance ranking can be unambiguously calculated for tournaments in which there are unrated players (players with no historical results). Thus, if there is an unrated player, we can assign him an arbitrarily chosen rating and this election does not affect the final ranking. The recursive performance proposes a way to divide the total strength of the tournament among the players. Therefore, when used as a rating method, there is neither inflation nor deflation with respect to the initial ratings.

\section{Robustness in $F$}

In order to apply the recursive performance in a given competitive environment, we first need to estimate the function $F$ that governs it. This estimation is subject to error and

\footnotetext{
${ }^{5}$ Most tournaments in disciplines such as chess and Othello have this property.

${ }^{6}$ Round-robin tournaments have a special structure and different approaches have been taken to define ranking methods within this family of tournaments. See for instance Daniels (1969), Stob (1985) and Chapter 6.1 in David (1988).
} 
hence we need to ensure that the ranking method is robust in $F$, that is, small changes of $F$ lead to small changes of the recursive performance. This follows immediately from the fact that the recursive performance is a solution of the linear system (11).

\section{Consistency with $F$}

Given two vectors of ratings $r^{1}, r^{2} \in \mathbb{R}^{n}$, we say that they are essentially identical for function $F$ if $F\left(r_{i}^{1}, r_{j}^{1}\right)=F\left(r_{i}^{2}, r_{j}^{2}\right)$ for all $i \neq j$. That is, two vectors are essentially identical if they lead to the same predictions for any given match between players in $N$. In linear paired comparisons analysis this is equivalent to the existence of $\lambda \in \mathbb{R}$ such that $r^{1}=r^{2}+\lambda e$.

Lemma 3. Let $(N, A)$ be a tournament and $F_{l}$ a linear rating function. Then, all the solutions of (1) are essentially identical for function $F_{l}$.

Proof. It follows from the fact that all the solutions of (1) are of the form $\hat{p}+\operatorname{span}\{e\}$.

Because of this property, we make a slight abuse of language and use the words recursive performance to encompass all the solutions of (11). Then, the total strength of the tournament suffices to pin a unique rating.

Now, we are ready to introduce a consistency property. We say that a rating $r \in \mathbb{R}^{n}$ is consistent with $F$ for tournament $(N, A)$ if the $r$-performance rating vector is essentially identical to $r$. This consistency property can also be extended to any ranking (not necessarily cardinal). A ranking $\succeq$ is consistent with $F$ for the tournament $(N, A)$ if there is a rating $r \in \mathbb{R}^{n}$ that is consistent with $F$ and whose induced ranking coincides with $\succeq$.

At the end of Section 6 we give a proof of the following result.

Proposition 4. Let $(N, A)$ be a tournament and $F_{l}$ a linear rating function. Then, the ranking induced by the recursive performance is the unique one that is consistent with $F_{l}$.

\section{$6 \quad$ Mathematical results}

In this section we prove the technical results we have used throughout Sections 4 and 5 . Although these results are stated for tournaments, they may be written just in terms of linear algebra. We follow Ciarlet (1989).

A linear iterative method is (globally) convergent if and only if the eigenvalues of the corresponding matrix are, in absolute value, less than 1 . For any tournament $(N, A)$ we have $\bar{M} e=e$ and thus 1 is an eigenvalue of $\bar{M}$. In this section we prove that, under the assumptions $\mathrm{A} 1$ and $\mathrm{A2}$, the iterated performance restricts to a vector subspace where the absolute values of the eigenvalues of $\bar{M}$ are less than 1 and, hence, the method converges.

Let $(N, A)$ be a tournament. For each $v, w \in \mathbb{R}^{n}$, we define $\langle v, w\rangle:=v^{t} D^{M} w=$ $\sum_{i=1}^{n} m_{i} v_{i} w_{i}$. Since $D^{M}$ is a diagonal matrix and $m_{i}>0$ for all $i,\langle\cdot, \cdot\rangle$ is an inner product in $\mathbb{R}^{n}$, which generalizes the Euclidean inner product. The former, which depends on the tournament, is referred to as the inner product associated with $(N, A)$.

If $(N, A)$ is a tournament, then $M$ is a symmetric matrix but $\bar{M}$ is not symmetric in general. However, there is a kind of symmetry in $\bar{M}$, namely, $\bar{M}_{i j}=0$ if and only if $\bar{M}_{j i}=0$. Motivated by this fact, we say that two matrices $B \in \mathcal{M}_{k \times l}$ and $C \in \mathcal{M}_{l \times k}$ are null-transpose if for each $i \in\{1, \ldots, k\}$, and each $j \in\{1, \ldots, l\}, B_{i j}=0$ if and only if $C_{j i}=0$. With a slight abuse of notation we denote by $B^{\text {nt }}$ a matrix that is null-transpose of $B$. Note that, although $B^{n t}$ is not unique, $B^{n t}=0$ if and only if $B=0$. 
Theorem 5. Let $(N, A)$ be a tournament and $\langle\cdot, \cdot \cdot\rangle$ its associated inner product. Then

(i) The matrix $\bar{M}$ is self-adjoint with respect to $\langle\cdot, \cdot\rangle$. Moreover, it is diagonalizable, its eigenvalues are real and the eigenspaces are orthogonal with respect to $\langle\cdot, \cdot\rangle$.

(ii) If $\lambda$ is an eigenvalue of $\bar{M}$, then $|\lambda| \leq 1$.

(iii) $(N, A)$ satisfies A1 if and only if the multiplicity of the eigenvalue 1 is one.

(iv) $(N, A)$ satisfies $\mathrm{A} 2$ if and only if -1 is not an eigenvalue of $\bar{M}$.

Proof. Let $(N, A)$ and $\left(N^{\prime}, A^{\prime}\right)$ be equivalent tournaments with matches matrices $M$ and $M^{\prime}$ respectively. Then, there exists $L \in \Pi_{n}$ such that $M=L M^{\prime} L^{-1}$. Clearly, $D^{M}=L D^{M^{\prime}} L^{-1}$ and thus $\bar{M}=L \bar{M}^{\prime} L^{-1}$. Hence, $\bar{M}$ and $\bar{M}^{\prime}$ are similar matrices and their eigenvalue structure is the same. Therefore, we may make, without loss of generality, any assumption regarding the ordering of the indices. We also recall that $\bar{M}_{i j} \geq 0$ for all $i, j \in\{1, \ldots, n\}$. For each $k \in \mathbb{N}$, we define $e^{k}:=(1, \ldots, 1) \in \mathbb{R}^{k}$. Note that $e^{n}=e$.

Claim (i): Since $M$ and $D^{M}$ are symmetric, $\langle v, \bar{M} w\rangle=v^{t} D^{M} \bar{M} w=v^{t} M w=w^{t} M v=$ $w^{t} D^{M} \bar{M} v=(\bar{M} v)^{t} D^{M} w=\langle\bar{M} v, w\rangle$. The second part follows from the spectral theorem.

Claim (ii): The matrix norm $\|\cdot\|_{\infty}$ is defined as $\|B\|_{\infty}:=\max _{i \in\{1, \ldots, n\}} \sum_{j=1}^{n}\left|B_{i j}\right|$ for any $B \in \mathcal{M}_{n \times n}$. By definition we have $\|\bar{M}\|_{\infty}=1$ and hence (ii) follows from Theorem 1.4-3 in Ciarlet (1989).

Claim (iii): Assume that $(N, A)$ does not satisfy A1. Then, $\bar{M}$ may be written as

$$
\bar{M}=\left(\begin{array}{c|c}
C & 0 \\
\hline 0 & E
\end{array}\right), \quad \text { with } C \in \mathcal{M}_{k \times k} \text { and } E \in \mathcal{M}_{l \times l} .
$$

Hence, $\bar{M}\left(e^{k} \mid 0\right)=\left(e^{k} \mid 0\right)$ and $\bar{M}\left(0 \mid e^{l}\right)=\left(0 \mid e^{l}\right)$. Thus, 1 has multiplicity at least 2 .

Conversely, assume that 1 has multiplicity greater than one. Then, since $\bar{M}$ is diagonalizable there exists $v \in \mathbb{R}^{n}$, linearly independent from $e$, such that $\bar{M} v=v$. Assume that $v_{1}=1$ and that the components of $v$ are decreasingly ordered, that is, $v_{i} \geq v_{j}$ for $i>j$. Let $k \in \mathbb{N}$ be such that $v_{k}=1$ and $v_{k+1}<1$. Since $v$ and $e$ are linearly independent, $k<n$ and $\bar{M}$ may be decomposed as

$$
\bar{M}=\left(\begin{array}{c|c}
C_{1} & E \\
\hline E^{n t} & C_{2}
\end{array}\right), \text { where } C_{1} \in \mathcal{M}_{k \times k}, C_{2} \in \mathcal{M}_{(n-k) \times(n-k)} \text { and } E \in \mathcal{M}_{k \times(n-k)} .
$$

Now, if $E$ has a nonzero row, namely row $i$, we get

$$
1=v_{i}=(\bar{M} v)_{i}=\sum_{j=1}^{k} \bar{M}_{i j}+\sum_{j=k+1}^{n} \bar{M}_{i j} v_{j}<\sum_{j=1}^{n} \bar{M}_{i j}=1
$$

contradiction. This proves $E=0$ and $E^{n t}=0$, which is a contradiction with A1,

Claim (iv): Assume $(N, A)$ does not satisfy $\mathrm{A} 2$. Then we may write $\bar{M}$ as

$$
\bar{M}=\left(\begin{array}{c|c}
0 & C \\
\hline E & 0
\end{array}\right), \quad \text { with } C \in \mathcal{M}_{k \times l} \text { and } E \in \mathcal{M}_{l \times k} .
$$

Hence, $\bar{M}\left(e^{k} \mid-e^{l}\right)=-\left(e^{k} \mid-e^{l}\right)$ and -1 is an eigenvalue of $\bar{M}$.

Conversely, assume that -1 is an eigenvalue of $\bar{M}$. Let $v \in \mathbb{R}^{n}$ be such that $\bar{M} v=-v$ and $1=v_{1} \geq v_{2} \geq \cdots \geq v_{n} \geq-1$. Again, there exists $k \in \mathbb{N}, k<n$ such that $v_{k}=1$ and $v_{k+1}<1$. Hence, $\bar{M}$ may be decomposed as in (2). 
We show that $C_{1}=0$. Let $i \in\{1, \ldots, k\}$. Since $\sum_{j=1}^{n} \bar{M}_{i j}=1$, we have $-1=-v_{i}=(\bar{M} v)_{i}=\sum_{j=1}^{k} \bar{M}_{i j}+\sum_{j=k+1}^{n} \bar{M}_{i j} v_{j} \geq \sum_{j=1}^{k} \bar{M}_{i j}+\left(1-\sum_{j=1}^{k} \bar{M}_{i j}\right)(-1)=2 \sum_{j=1}^{k} \bar{M}_{i j}-1$.

Hence, $\sum_{j=1}^{k} \bar{M}_{i j} \leq 0$. Since $\bar{M}_{i j} \geq 0$, the $i$ th row of $C_{1}$ is zero. Therefore, $C_{1}=0$.

Note that $v_{n}=-1$ since, otherwise, taking $i \in\{1, \ldots, k\}$ we get $-1=-v_{i}=(\bar{M} v)_{i}=$ $\sum_{j=k+1}^{n} \bar{M}_{i j} v_{j}>-1$. Let $l \in \mathbb{N}$ be such that $v_{n-l}>-1$ and $v_{j}=-1$ for $j \geq n-l$. Clearly, $l>0$. Then, we may further decompose $\bar{M}$ as

$$
\bar{M}=\left(\begin{array}{c|c|c}
0 & E_{1} & E_{2} \\
\hline E_{1}^{n t} & C_{21} & C_{22} \\
\hline E_{2}^{n t} & C_{22}^{n t} & C_{23}
\end{array}\right) \text { with } E=\left(E_{1} \mid E_{2}\right), C_{2}=\left(\begin{array}{c|c}
C_{21} & C_{22} \\
\hline C_{22}^{n t} & C_{23}
\end{array}\right) \text { and } E_{2} \in \mathcal{M}_{k \times l} .
$$

If $k+l=n$, then this second decomposition is trivial $\left(E=E_{2}\right.$ and $\left.C_{2}=C_{23}\right)$. Otherwise, we claim that $E_{1}=0$. If the $i$ th row of $E_{1}$ is nonzero, then there is $j \in\{k+1, \ldots, n-l\}$ such that $v_{j}>-1$. Hence, $-1=-v_{i}=(\bar{M} v)_{i}=\sum_{j=k+1}^{n} \bar{M}_{i j} v_{j}>-1$, contradiction. Therefore, $E_{1}=0$.

Now, we show that $\left(C_{22}^{n t} \mid C_{23}\right)$ is zero. If the $i$ th row of $\left(C_{22}^{n t} \mid C_{23}\right)$ is nonzero, then there is $j \in\{k+1, \ldots, n\}$ such that $v_{j}<1$. Hence, $1=-v_{i}=(\bar{M} v)_{i}=\sum_{j=1}^{n} \bar{M}_{i j} v_{j}<1$, contradiction. Therefore, the above decomposition reduces to

$$
\bar{M}=\left(\begin{array}{c|c|c}
0 & 0 & E_{2} \\
\hline 0 & C_{21} & 0 \\
\hline E_{2}^{n t} & 0 & 0
\end{array}\right)
$$

which contradicts $\mathrm{A}^{7}$

Corollary 6. Let $(N, A)$ be a tournament satisfying assumptions A1 and A2. Then,

$$
\lim _{l \rightarrow \infty} \bar{M}^{l}=\frac{1}{\sum_{i=1}^{n} m_{i}}\left(\begin{array}{ccc}
m_{1} & \cdots & m_{n} \\
\vdots & & \vdots \\
m_{1} & \cdots & m_{n}
\end{array}\right) .
$$

Proof. Let $v \in \mathbb{R}^{n}$. By Theorem 5 there exists a basis of eigenvectors $\left\{w_{1}=e, w_{2}, \ldots, w_{n}\right\}$, orthogonal with respect to the inner product associated with $(N, A)$, which we denote by $\langle\cdot, \cdot\rangle$. For each $i \in\{1, \ldots, n\}$, let $\lambda_{i}$ be the eigenvalue associated with $w_{i}$. Now, it suffices to show that $\left(\lim _{l \rightarrow \infty} \bar{M}^{l}\right) v=\left(\frac{1}{\operatorname{tr} D^{M}} e e^{t} D^{M}\right) v$ for all $v \in \mathbb{R}^{n}$. For each $i \in\{1, \ldots, n\}$, we have $\left\langle\left(\lim _{l \rightarrow \infty} \bar{M}^{l}\right) v, w_{i}\right\rangle=\lim _{l \rightarrow \infty}\left\langle v, \bar{M}^{l} w_{i}\right\rangle=\left(\lim _{l \rightarrow \infty} \lambda_{i}^{l}\right)\left\langle v, w_{i}\right\rangle$. Since $\lambda_{1}=1$ and $\left|\lambda_{i}\right|<1$ for each $i \in\{2, \ldots, n\}$, we get $\left(\lim _{l \rightarrow \infty} \bar{M}^{l}\right) v=(\langle v, e\rangle /\langle e, e\rangle) e$. By definition of $\langle\cdot, \cdot\rangle$, we have $\langle e, e\rangle=\operatorname{tr} D^{M}$ and $\langle v, e\rangle=e^{t} D^{M} v$, from where the result follows.

Corollary 7. Let $(N, A)$ be a tournament satisfying A1 and A2, Let $\langle\cdot, \cdot\rangle$ be its associated inner product. Then, for all $l \in \mathbb{N},\left\langle\hat{p}^{(l)}, e\right\rangle=\langle r, e\rangle$, or equivalently, $\sum_{i} m_{i} \hat{p}_{i}^{(l)}=$ $\sum_{i} m_{i} r_{i}=\sigma^{r}$.

Proof. Recall that $\bar{M}$ is self-adjoint with respect to $\langle\cdot, \cdot\rangle$ by Theorem 5 (i). By Corollary 6 , $\lim _{l \rightarrow \infty} \bar{M}^{l}$ exists and $\left(\lim _{l \rightarrow \infty} \bar{M}^{l}\right) c=\left(\left(\sum_{i} m_{i} c_{i}\right) /\left(\sum_{i} m_{i}\right)\right) e$. Hence, $\langle\hat{c}, e\rangle=\langle c, e\rangle-$ $\left\langle\left(\lim _{l \rightarrow \infty} \bar{M}^{l}\right) c, e\right\rangle=\langle c, e\rangle-\lim _{l \rightarrow \infty}\left\langle c, \bar{M}^{l} e\right\rangle=0$. Then, $\left\langle\hat{p}^{(0)}, e\right\rangle=\langle r, \bar{M} e\rangle+\langle\hat{c}, e\rangle=\langle r, e\rangle$. By induction, $\left\langle\hat{p}^{(l)}, e\right\rangle=\left\langle\hat{p}^{(l-1)}, \bar{M} e\right\rangle+\langle\hat{c}, e\rangle=\langle r, e\rangle$.

\footnotetext{
${ }^{7}$ Note that, if the latter decomposition happens to be nontrivial (presence of $C_{21}$ ), we also violate A1
} 
We are now ready to prove the main result of this paper.

Proof of Theorem 圆. Defining $q^{(l)}:=\hat{p}^{(l)}-r$ for $l \geq 0$ we have the equivalent iterative method $q^{(0)}=\bar{M} r+\hat{c}-r$ and $q^{(l)}=\bar{M} q^{(l-1)}+q^{(0)}, l \in \mathbb{N}$. Let $\langle\cdot, \cdot\rangle$ be the inner product associated with $(N, A)$. By Corollary $7,\left\langle q^{(l)}, e\right\rangle=0$ for all $l \in \mathbb{N}$. Therefore, the iterative method restricts to the vector subspace $e^{\perp}$. By Theorem [5 the absolute values of the eigenvalues of $\bar{M}_{\mid e^{\perp}}$ are smaller than 1. Hence, the iterative method converges (Ciarlet, 1989).

Proof of Proposition 4 Let $\succeq$ be a ranking consistent with $F_{l}$. Then, there exists a rating $r \in \mathbb{R}^{n}$ and a constant $\lambda \in \mathbb{R}$ such that $r=p^{r}+\lambda e=\bar{M} r+c+\lambda e$. Taking inner product with $e$ (as in Corollary 6) we get $\langle r, e\rangle=\langle r, e\rangle+\langle c, e\rangle+\lambda\langle e, e\rangle$. Hence, $\lambda=-\langle c, e\rangle /\langle e, e\rangle$ and thus $c+\lambda e=\hat{c}$. This implies that $r$ is a solution of (11) and the result follows.

\section{References}

AmiR, R. (2002): "Impact-Adjusted Citations as a Measure of Journal Quality," Tech. rep., CORE Discussion Paper 2002/74.

Bradley, R. A. AND M. E. Terry (1952): "Rank analysis of incomplete block designs. I. The method of paired comparisons," Biometrika, 39, 324-345.

Ciarlet, P. G. (1989): Introduction to numerical linear algebra and optimisation, Cambridge: Cambridge University Press.

Daniels, H. (1969): "Round-Robin Tournament Scores," Biometrika, 56, 295-299.

David, H. A. (1988): The method of paired comparisons, London: Chapman and Hall.

GodDARD, S. T. (1983): "Ranking in tournaments and group decision making," Management Science, 29, 1384-1392.

Iqbal, A., W. Cook, And M. Kress (1986): "On the minimum violations ranking of a tournament," Management Science, 32, 660-672.

Kendall, M. G. And B. B. Smith (1940): "On the method of paired comparisons," Biometrika, 31, 324-345.

LASLIER, J. (1997): Tournament Solutions and Majority Voting, Springer-Verlag. Berlin.

Liebowitz, S. AND J. PALmer (1984): "Assesing the Relative Impacts of Economic Journals," Journal of Economic Literature, 22, 77-88.

Moon, J. W. (1968): Topics on Tournaments, New York.

Palacios-Huerta, I. And O. VoliJ (2004): "The Measurement of Intellectual Influence," Econometrica, 72, 963-977.

Rubinstein, A. (1980): "Ranking the Participants in a Tournament," SIAM Journal on Applied Mathematics, 38, 108-111.

Slutzki, G. And O. VoliJ (2005): "Ranking Participants in Generalized Tournaments," International Journal of Game Theory, 33, 255-270. 
Sтов, M. (1985): "Rankings from Round Robin tournaments," Management Science, 31, 1191-1195.

Zermelo, E. (1929): "Die Berechnung der Turnier-Ergebnisse als ein Maximumproblem der Wahrscheinlichkeitsrechnung," Mathematische Zeitschrift, 29, 436-460. 